\title{
On phase, action and canonical conservation laws in kinematic-wave theory
}

\author{
Gerard A. Maugin \\ Université Pierre et Marie Curie (Paris-6), Institut Jean Le Rond d'Alembert \\ UMR 7190 CNRS, Case 162, 4 place Jussieu, 75252 Paris Cedex 05, France \\ E-mail : gam@ccr.jussieu.fr
}

Received November 26, 2007

\begin{abstract}
Canonical equations of energy and momentum are constructed in the kinematic-wave theory of waves in a continuum. This is done in analogy with what is achieved in nonlinear continuum mechanics. The starting point is a generalized balance of wave action. The standard formulas are recovered when the system follows from the averaged-Lagrangian variational formulation of Whitham.
\end{abstract}

PACS: $75.40 . \mathrm{Gb}$ Dynamic properties (dynamic susceptibility, spin waves, spin diffusion, dynamic scaling, etc.); 05.45.-a Nonlinear dynamics and chaos.

Keywords: waves, phase, action, momentum, conservation laws.

\section{Introduction}

During the last two decades many works following a line opened by J.D. Eshelby have set forth the essential role played by conservation laws issued from the application of Noether's theorem in modern continuum mechanics (see, e.g., Maugin $[1,2]$ ), especially in the study of the evolution of singularity sets in crystal elasticity (dislocations, cracks, phase-transition fronts) where components of the divergence of the so-called energy-momentum tensor intervening in the conservation (or non strict conservation) of canonical momentum provide the driving force on these «objects». Until recently, it was thought that this could be formulated only for conservative systems derivable from a Lagrangian (hence the application of Noether's theorem). But in recent works the author has shown how the canonical equations of momentum and energy could be constructed systematically even in a general dissipative framework exhibiting both intrinsic dissipation and heat conduction (e.g., Maugin $[3,4]$ ). Remember that these two equations are related to the system's invariance (or lack of invariance) with respect to space-time translations, space being parametrized by the material coordinates $\mathbf{X}$ of finite-strain continuum mechanics and time $t$ is Newton's time (to remain in a non relativistic framework).

Of course one is tempted to examine what happens with the wavelike quantities usually associated in lin- ear-wave mechanics, i.e., material wave vector $\mathbf{K}$ and frequency $\omega$ since these combine with $\mathbf{X}$ and $t$ in a duality exhibited by the usual phase function $\varphi=\mathbf{K} \cdot \mathbf{X}-\omega t$. The use of generally defined wave number and frequency by Lighthill [5], Whitham [6] and others led to the concept of kinematic-wave theory which in turn has provided astute ways to deal with certain problems of nonlinear wave propagation (in particular, for weakly nonlinear and dispersive systems yielding nonlinear Schrödinger equations and the notions of bright and dark solitons) in the expert hands of authors such as Benney [7] and Newell [8]. We have produced a rare but aesthetically pleasing illustration in the case of surface solitons [9]. Kinematic-wave theory is discussed and illustrated in books (Whitham [10], Maugin [11], Ostrovsky and Potapov [12]).

In recent works, we naturally pondered the transcription of Eshelby's ideas in this general nonlinear-wave framework. At first we again applied this only to the conservative case, along with a due application of Noether's theorem (Maugin $[13,14]$ ). Going one step further in the present paper, we give the expression of canonical equations for wave-kinematics for general systems since waves also propagate in inhomogeneous dissipative continua including even source terms. Accordingly, a rarely considered notion in continuum mechanics, that of $a c$ tion, is introduced and plays a fundamental role. 


\section{Generalized phase function}

A general smooth motion of a continuum in Euclidean-Newtonian space-time is represented by the smooth vector-valued function $\mathbf{x}=\overline{\mathbf{x}}(\mathbf{X}, t)$, where $\mathbf{x}$ stands for the actual position and $\mathbf{X}$ represents the set of material coordinates on the material manifold [1]. The phase of a plane linear wave in a continuum is defined in this material description by

$$
\varphi(\mathbf{X}, t)=\tilde{\varphi}(\mathbf{K}, \omega)=\mathbf{K} \cdot \mathbf{X}-\omega t,
$$

where $\mathbf{K}$ is the material wave vector and $\omega$ is the associated circular frequency. But in the kinematic-wave theory a general phase function

$$
\bar{\varphi}=\bar{\varphi}(\mathbf{X}, t)
$$

is introduced from which the material wave vector $\mathbf{K}$ and the frequency $\omega$ are defined by

$$
\mathbf{K}=\frac{\partial \bar{\varphi}}{\partial \mathbf{X}}=\nabla_{R} \bar{\varphi}, \quad \omega=-\frac{\partial \bar{\varphi}}{\partial t} .
$$

Whence there follows at once the two equations (curl-free nature of $\mathbf{K}$, and conservation of wave vector)

$$
\begin{gathered}
\nabla_{R} \times \mathbf{K}=0, \\
\frac{\partial \mathbf{K}}{\partial t}+\nabla_{R} \omega=0 .
\end{gathered}
$$

In particular, (3) are trivially satisfied for plane wave solutions for which the last of (1) holds true. For an inhomogeneous rheonomic linear behavior with dispersion we have the dispersion relation

$$
\omega=\Omega(\mathbf{K} ; \mathbf{X}, t) .
$$

Accordingly, the conservation of wave vector (5) becomes

$$
\frac{\partial \mathbf{K}}{\partial t}+\mathbf{V}_{g} \cdot \nabla_{\mathrm{R}} \mathbf{K}=-\left.\frac{\partial \Omega}{\partial \mathbf{X}}\right|_{\operatorname{expl}}, \quad \mathbf{V}_{g}=\frac{\partial \Omega}{\partial \mathbf{K}},
$$

and thus the Hamiltonian system [15]

$$
\frac{D \mathbf{X}}{D t}=\frac{\partial \Omega}{\partial \mathbf{K}}, \quad \frac{D \mathbf{K}}{D t}=-\left.\frac{\partial \Omega}{\partial \mathbf{X}}\right|_{\text {expl }},
$$

where we have set

$$
\frac{D}{D t} \equiv \frac{\partial}{\partial t}+\mathbf{V}_{g} \cdot \nabla_{R}
$$

Simultaneously, we have the Hamilton-Jacobi equation (compare eq. (3))

$$
\frac{\partial \varphi}{\partial t}+\Omega\left(\mathbf{X}, t ; \mathbf{K}=\frac{\partial \varphi}{\partial \mathbf{X}}\right)=0 .
$$

If we now consider a wave in an inhomogeneous rheonomic dispersive nonlinear material, the frequency will also depend on the amplitude. Let a the $n$-vector of $R^{n}$
$R^{n}$ that characterizes this small slowly varying amplitude of a complex system (in general with several degrees of freedom). Thus, now,

$$
\omega=\Omega(\mathbf{K} ; \mathbf{X}, t, \mathbf{a}) .
$$

Accordingly, the second of Hamilton's equations (7) will now read [15]

$$
\frac{D \mathbf{K}}{D t}=-\left.\frac{\partial \Omega}{\partial \mathbf{X}}\right|_{\text {expl }}+\mathbf{A} \cdot\left(\nabla_{\mathrm{R}} \mathbf{a}\right)^{T}, \quad \mathbf{A}:=-\frac{\partial \Omega}{\partial \mathbf{a}} .
$$

In the studies of Newell [8] and Maugin and Hadouaj [9], one is even led to considering a nonlinear «dispersive» dispersion relation in which the assumed slowly varying quantities such as space and time derivative of the amplitude are involved in the function $\Omega$, which relation becomes a true «wave equation» itself for the amplitude.

\section{Generalized action function}

In full similarity with (1), the scalar quantity called the action density per unit reference volume is classically defined by

$$
S(\mathbf{X}, t)=\widetilde{S}(\mathbf{P}, H)=\mathbf{P} \cdot \mathbf{X}-H t,
$$

where $\mathbf{P}$ is a material momentum and $H$ is an energy (Hamiltonian). It is on the basis of this and the invariance of $S$ that L. de Broglie deduced his celebrated relation $\mathbf{P}=\hbar \mathbf{K}$, if Planck's relation $H=\hbar \omega$ applies, so that $S=\hbar \varphi$ in this essentially linear theory (here $\hbar$ is Planck's reduced unit of action). Forgetting about the expression given in (13), consider a general smooth function $S=\bar{S}(\mathbf{X}, t)$ and define general material momentum and energy by (compare to (3))

$$
\mathbf{P}=\nabla_{R} \bar{S}, H=-\left.\frac{\partial \bar{S}}{\partial t}\right|_{X},
$$

where $\nabla_{R}$ is the material gradient and $d / d t:=\partial /\left.\partial t\right|_{X}$ is the material time derivative. Obviously then,

$$
\nabla_{R} \times \mathbf{P}=0, \quad \frac{d \mathbf{P}}{d t}=-\nabla_{R} H .
$$

From the second of these it follows that if the first is valid initially, it remains valid in time. The case (13) satisfies (14) trivially. In standard analytical mechanics, the first of (14) is none other than the Jacobi equation of motion; it would be de Broglie's «guidance» equation in the causal interpretation of quantum mechanics; see post scriptum below.

In nonlinear (of course conservative) dynamic inhomogeneous (but scleronomic $=$ no explicit time dependence) elasticity we know the expression of $H$, for instance as a sufficiently regular function 


$$
H=\bar{H}(\mathbf{P}, \mathbf{X}, \nabla \mathbf{X}, N),
$$

where $\nabla \mathbf{X}=\mathbf{F}^{-1}$ is the spatial gradient of the «inverse motion» [1,2], and $N$ is the entropy density. As a matter of fact, we more precisely have

$$
H=\frac{1}{2 \rho_{0}} \mathbf{P} \cdot \mathbf{C}^{-1} \cdot \mathbf{P}+E\left(\mathbf{X}, \mathbf{F}^{-1}, N\right),
$$

where $\rho_{0}$ is the reference (possibly $\mathbf{X}$ dependent) matter density, $E$ is the internal energy per reference volume, $\mathbf{P}=\rho_{0} \mathbf{C} \cdot \mathbf{V}, \mathbf{C}=\mathbf{F}^{T} \cdot \mathbf{F}$, and $\mathbf{V}=-\mathbf{F}^{-1} \cdot \mathbf{v}$, with $\mathbf{v}=\partial \overline{\mathbf{x}} / \partial t$, $\mathbf{F}=\nabla_{R} \overline{\mathbf{x}}$. This is not the standard formulation of elasticity, but the one using the so-called «inverse motion». We let the reader do the tricky exercise that the second of (15) then yields the canonical equations of energy and momentum in the form (cf. [1]):

$$
\frac{d N}{d t}=0, \frac{d \mathbf{P}}{d t}=\operatorname{div}_{R} \mathbf{b}+\mathbf{f}^{\text {inh }},
$$

wherein

$$
\begin{gathered}
\mathbf{b}=-(L \mathbf{1}+\mathbf{T} \cdot \mathbf{F}), \quad L=\mathbf{P} \cdot \mathbf{V}-H=K-E, \\
\mathbf{T}=\partial \bar{E} / \partial \mathbf{F}, \quad \mathbf{f}^{\text {inh }}=\left.\frac{\partial L}{\partial \mathbf{X}}\right|_{\text {expl }},
\end{gathered}
$$

where the explicit gradient in the last quantity extracts the $\mathbf{X}$ explicit dependence of the «Lagrangian» $L$ in the case of material inhomogeneity via the density and the internal energy. Tensor $\mathbf{b}$ is called the material Eshelby stress tensor. Had we started from a Lagrangian variational principle, equations (18) would have followed in this very form after application of Noether's theorem under time and (material) space translations. Works $[3,4]$ have shown how to establish the generalizations of (18) for a general continuum in the presence of dissipation and all types of additional effects.

Remark. The action is seldom considered as a basic quantity in continuum mechanics. However, a conservation-like equation is obtained for this action - as defined in (13) - for a group of simultaneous space and time transformations (expansions or scaling) as shown by the author [1] (Chapter 4) and also Lazar [16].

\section{Canonical energy equation for wave mechanics}

Imagine that we have to start with a local scalar balance law of the form

$$
\frac{d S}{d t}-\nabla_{R} \cdot \mathbf{W}=E_{d}
$$

Later on $S$ will be identified with an action, and $\mathbf{W}$ as an action flux, while $E_{d}$ is an external energy input. For the time being we formally multiply both sides of (20) by $\omega$, a circular frequency, and subtract from both sides of the resulting equation the quantity $d \widetilde{L} / d t$, where the scalar quantity $\widetilde{L}$ will be specified later. After some manipulation we obtain thus the formal equation

$\frac{d}{d t}(\omega S-\widetilde{L})-\nabla_{R} \cdot(\omega \mathbf{W})=h:=E_{d} \omega+S \frac{\partial \omega}{\partial t}+\mathbf{W} \cdot \nabla_{R} \omega-\frac{d \widetilde{L}}{d t}$.

Because of the meaning granted to $E_{d}$ this is an equation of energy balance. In particular, if we were working in the kinematic-wave theory of Lighthill and Whitham, $\widetilde{L}$ would be the so-called averaged (over the phase) Lagrangian such that $[10,13,14]$

$$
S=\frac{\partial \widetilde{L}}{\partial \omega}, \quad \mathbf{W}=\frac{\partial \widetilde{L}}{\partial \mathbf{K}}, \quad \widetilde{L}=\widetilde{L}(\mathbf{K}, \omega, \mathbf{X}, t),
$$

for an inhomogeneous rheonomic system. Then (21) yields the energy balance as

$$
\frac{d \widetilde{H}}{d t}-\nabla_{R} \cdot \widetilde{\mathbf{Q}}=\widetilde{h}:=h^{t}+h^{\mathrm{ext}},
$$

wherein energy density, effective heat flux and internal and external energy sources are given by

$$
\widetilde{H}=\omega S-\widetilde{L}, \quad \widetilde{\mathbf{Q}}=\omega \mathbf{W}, \quad h^{t}=-\left.\frac{\partial \widetilde{L}}{\partial t}\right|_{\text {expl }}, h^{\mathrm{ext}}=E_{d} \omega .
$$

In the absence of external heat source, (23) follows from the variational formulation of Whitham of the averaged Lagrangian after application of Noether's theorem for time translations.

\section{Canonical momentum equations for wave mechanics}

We proceed just like in the previous paragraph but multiply both sides of the a priori set equation (20) by a material (co-)vector which is none other than a wave vector $\mathbf{K}$ and add to both sides of the resulting vectorial equation the material co-vector $\nabla_{R} \widetilde{L}$. After some manipulations we arrive at the following co-vectorial balance equation

$$
\frac{d \widetilde{\mathbf{P}}}{d t}-\operatorname{div}_{R} \widetilde{\mathbf{b}}=\mathbf{f}:=E_{d} \mathbf{K}-S \nabla_{R} \omega-\mathbf{W} \cdot \nabla_{R} \cdot \mathbf{K}+\nabla_{R} \widetilde{L},
$$

where we have set

$$
\widetilde{\mathbf{P}}=S \mathbf{K}, \widetilde{\mathbf{b}}=-(\widetilde{L} \mathbf{1}-\mathbf{W} \otimes \mathbf{K}) .
$$

If $S$ is the action, then the first of the last two equations defines the material wave momentum and is a continuum generalization of de Broglie's relation. The mixed material tensor defined in (26) we called the material wave Eshelby tensor. If we are within the framework of the Lighthill-Whitham theory of the averaged Lagrangian $\widetilde{L}$, then equations (22) apply and computing the material gradient of $\widetilde{L}$ and substituting in the right-hand side of (25) 
we arrive at the balance of material wave momentum in the form

$$
\frac{d \widetilde{\mathbf{P}}}{d t}-\operatorname{div}_{R} \widetilde{\mathbf{b}}=\mathbf{f}^{\text {inh }}+\mathbf{f}^{\text {ext }},
$$

with «material forces» of inhomogeneity and external origin given by

$$
\mathbf{f}^{\text {inh }}=\left.\frac{\partial \widetilde{L}}{\partial \mathbf{X}}\right|_{\text {expl }}, \quad \mathbf{f}^{\text {ext }}=E_{d} \mathbf{K} .
$$

In the absence of external heat source, (27) follows from the variational formulation of Whitham of the averaged Lagrangian after application of Noether's theorem for material space translations.

\section{Amplitude dependence}

If we are in the strict framework of the averaged Lagrangian variational method, even supposing from the beginning that the averaged Lagrangian depends explicitly on the amplitude a of the studied wave process (as supposed in the generalized dispersion relation (11)), it is shown that one of Euler-Lagrange in fact results in the equation of amplitude independence

$$
0=\partial \widetilde{L} / \partial \mathbf{a} .
$$

unless, of course, there exists an external source $\mathbf{f}^{\mathbf{a}}$ balancing this. In this case equations (23) and (27) will contain additional terms in their right hand side, given by

$$
h^{\mathbf{a}}=\mathbf{f}^{\mathbf{a}} \cdot \dot{\mathbf{a}}, \mathbf{f}^{\text {ext. } \mathbf{a}}=\mathbf{f}^{\mathbf{a}} \cdot\left(\nabla_{R} \mathbf{a}\right)^{T} .
$$

\section{Conclusion}

Of course there are essential differences between the elasticity case recalled at the end of Section 3 and the wave case of Section 4. In the elasticity case, the departure point in the absence of variational formulation is the local balance of physical linear momentum, i.e., the basic balance law of continuum mechanics written in the actual configuration and involving the Cauchy stress or the first Piola-Kirchhoff stress T, along with a statement of the first law of thermodynamics [4]; and all agree on these. Furthermore, the nonlinear case and the case involving characteristic internal length scales (hence a weak nonlocality), such as in so-called gradient elasticity, are automatically included $[1,16]$ and prove extremely useful for studying directly nonlinear waves in crystals $[11,17]$. In the kinematic-wave theory briefly discussed in this paper, equation (21) is hardly conceived as a basic equation, although this is not forbidden but a little farfetched and we do not clearly see what nonlinearity and nonlocality mean. For sure, however, the momentum equation (25) should yield the time evolution of Brenig's wave momentum [18] since, for instance, $\widetilde{P}=\omega K a^{2}$ (up to a factor of modulus one) for a one-dimensional harmonic wave-like motion (see equation (6.3) in [14])

P.S. Some of the above- made considerations may be close to those found in the causal re-interpretation of quantum mechanics; see Jammer [19] Sections 2.5 and 2.6; Holland [20] Chapter 2.

1. G.A. Maugin, Material Inhomogeneities in Elasticity, Chapman and Hall, London (1993).

2. G.A. Maugin, Trans. ASME Appl. Mech. Rev. 48, 213 (1995).

3. G.A. Maugin, Mech. Res. Commun. 33, 705 (2006).

4. G.A. Maugin, Arch. Appl. Mech. 75, 723 (2006).

5. J.M. Lighthill, J. Inst. Maths. Applics. 1, 269 (1965).

6. G.B. Whitham, J. Fluid Mech. 22, 273 (1965).

7. D.J. Benney and A.C. Newell, J. Math. and Phys. 46, 133 (1967).

8. A.C. Newell, Solitons in Mathematics and Physics, S.I.A.M., Philadelphia (1985).

9. G.A. Maugin and H. Hadouaj, Phys. Rev. B44, 1266 (1992).

10. G.B. Whitham, Linear and Nonlinear Waves, Interscience-John Wiley, New York (1974).

11. G.A. Maugin, Nonlinear Waves in Elastic Crystals, Oxford University Press, Oxford (1999).

12. L.A. Ostrovsky and A.I. Potapov, Modulated Waves, Johns Hopkins University Press, Baltimore (1999).

13. G.A. Maugin, Wave Motion 44, 472 (2007).

14. G.A. Maugin, J. Phys. Conf. Series 62, 72 (2007).

15. G.A. Maugin, Proc. Est. Acad. Sci. Phys. Math. 52/1, 5 (2003).

16. M. Lazar and C. Anastassiadis, J. Elasticity 88, 5 (2007).

17. A.M. Kosevich, The Crystal Lattice : Phonons, Solitons, Dislocations, Berlin-Wiley-VCH (1999).

18. W. Brenig, Zeit. Phys. 143, 168 (1955).

19. M. Jammer, The Philosophy of Quantum Mechanics, J. Wiley-Science, New York (1974).

20. P.R. Holland, The Quantum Theory of Motion, Cambridge University Press, Cambridge, UK (1993). 